\title{
Planar motion controller design for a modular mechatronic device with heading compensation
}

\author{
Stefan Ristevski, Melih Cakmakci* \\ Department of Mechanical Engineering Bilkent University, Ankara 06800, Turkey
}

\section{A R T I C L E I N F O}

\section{Keywords:}

Motion control

Friction compensation

Mobile robots

\begin{abstract}
A B S T R A C T
MechaCells are designed as closed, scalable and modular semi-autonomous devices that can be used alone or part of a pack. In this paper, we discuss a locomotion system that uses the reaction force produced by a rotating unbalance that moves in a spherical domain with a steering mechanism. In order to produce the precise motion capability, a multi-loop controller is developed. This controller uses a friction compensation algorithm based on the mathematical model of the locomotion system. To improve the accuracy of tracking, conventional LuGre friction estimation model is extended for rapid directional changes of the MechaCell during planar motion. The linear and rotational acceleration of the device is also included in controller calculations since it affects the locomotion force generated by the unbalanced mass. The resulting control system is validated both with simulations and experiments and the effectiveness of the extended model and the controller is verified. Our results show significant improvement when a detailed friction compensation observer is used in the controller that includes the effect of sudden steering changes for precise path following.
\end{abstract}

\section{Introduction}

In recent years, there has been increased use of sensors and actuators and devices with on-board diagnostics that create useful controller design opportunities as reported in [1-4]. Due to their multi-domain nature, design and manufacturing of these smart mechatronic systems can be a challenging and a complicated task. One way to manage the complexities is to use modularity during the design and manufacturing phases as discussed in $[5,6]$. For example by increasing the componentsharing modularity, complicated systems can be built by using simple common modules that are identical in engineering properties.

The application of component-sharing modularity in mechatronic systems, especially in the ground robotics field, is studied by various researchers. In [7], an overview of the reconfigurable modular systems is given. Building scalable, self-repairing, self-sustaining and selfconfiguring systems are presented as grand challenges in this field. A design method and experiments for whole-body locomotion by a modular robot is presented in [8]. In this work, the locomotion of the whole structure is studied rather than the module motion. In [9], a modular robotic device was designed such that modules can self-assemble themselves to form a robotic structure, and can move independently. However, the locomotion system used in this module is not enclosed, which may be problematic for precision motion and for un-ideal surface conditions. Generally, the final external structure of the system is studied rather than how to unify the capabilities and strengthen device collaboration. In [10], a modular self configurable robot that changes shape horizontally to convey micro parts is developed, which is also close to the application used in this paper.

Our motivation is to apply the principle of component-sharing modularity and utilize its potential in mechatronics area by developing a scalable mechatronic module that can be used as the building block for more complicated systems. Component sharing modularity in mechatronic systems imply that, complex systems can be build from a single module. This module should be miniaturizable, have the ability of both sensing and actuation and should be strong/sturdy enough to address the external disturbance such as surface friction. Similar examples in recent literature is the research where the swarm devices such as Kilobot [11], Alice robots [12] were developed. However, on contrary to swarm devices, mechatronic modules discussed here are designed to accomplish tasks that involve physical interaction as part of a larger system with controlled actuation and sensing. These devices will be shortly referred as MechaCells.

In Fig. 1, a precision positioning task performed by MechaCells is illustrated as an example. A group of Mechacells can position a workpiece on a plane so that a laser machining operation is performed. This type of handling can be important especially for the cases when the workpiece is too fragile to be attached inside a fixture on a positioning system. Moreover, this operation may need to be done at a hard to reach

\footnotetext{
This paper was recommended for publication by Associate Editor Prof. Paolo Rocco.

* Corresponding author.

E-mail address: melihc@bilkent.edu.tr (M. Cakmakci).
} 


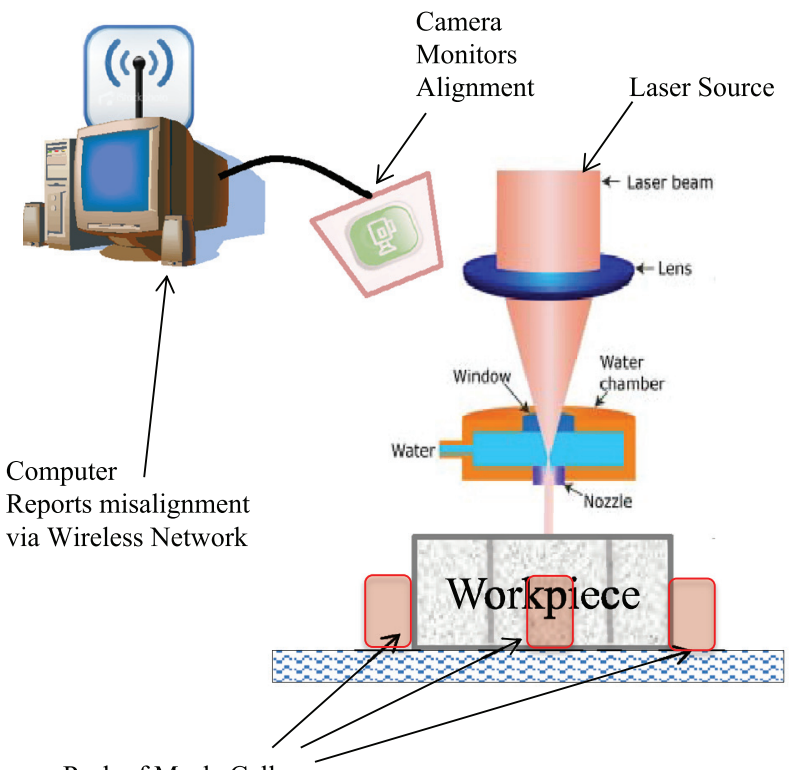

Pack of MechaCells move the Workpiece while the piece is machined

\section{Part (a) Side View of the Machining Testbed}

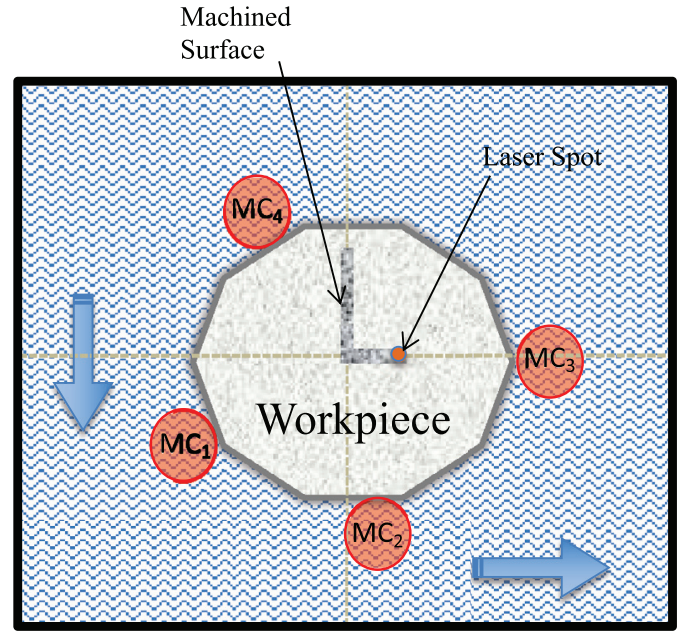

\section{Part (b) Top View From the Camera during Laser Machining.}

Fig. 1. A small scale laser manufacturing system illustrating the use of MechaCell devices to guide a workpiece on a planar surface.
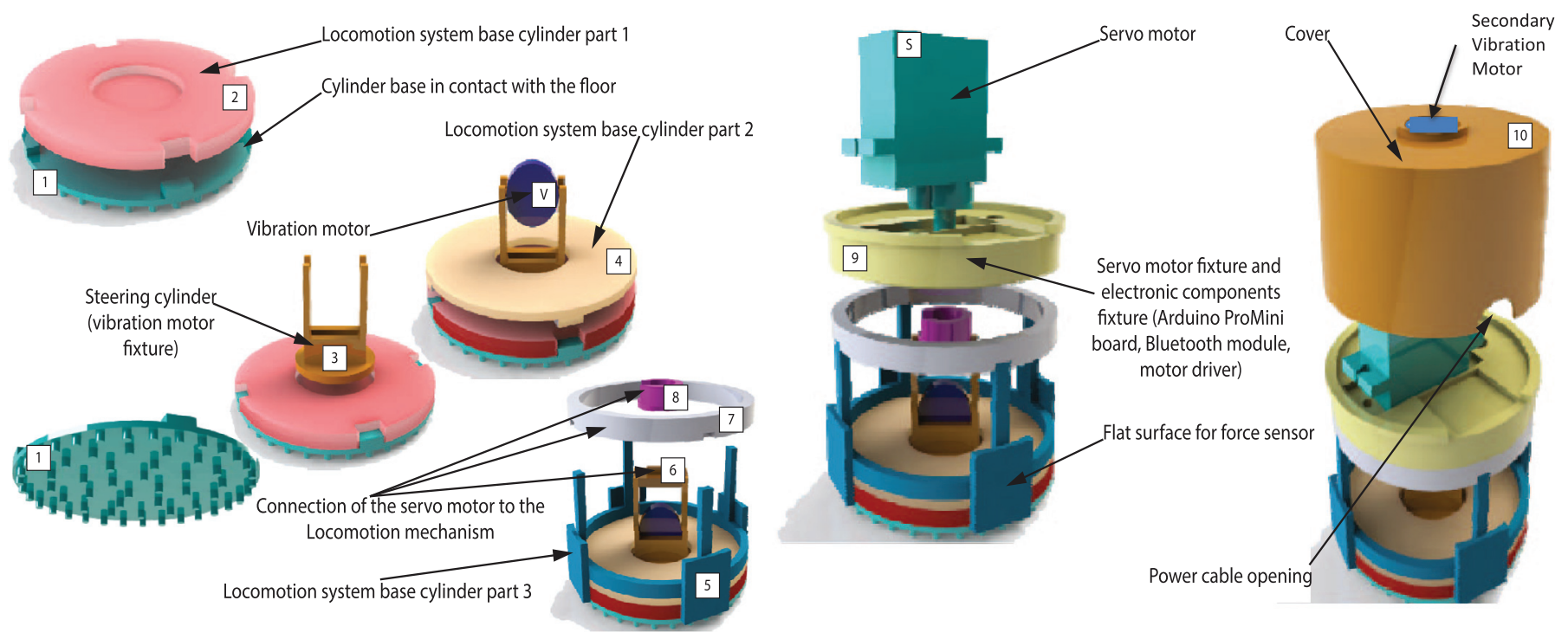

Fig. 2. Parts of the MechaCell and assembly steps.

area such as in the cases of on-board repairs. The example depicted in Fig. 1 suggests that the mechatronic systems with more precise tasks will require modules that have advanced capabilities such as controlled motion and force.

In Fig. 2, the mechatronic module, which was designed and developed to test the idea of applying component-sharing modularity (i.e. building systems from common modules) for generic systems, is shown. The numbers in this figure show the assembly order. The mechanical design of this device and its components were discussed in detail in
$[13,14]$. In [15], the cooperative operation strategies for MechaCells is discussed for dextereous manipulation. This device contains a steering servo-motor (S), a primary vibration motor (V), a three axis accelerometer and a surface pressure sensor and finally an embedded computer with wireless communication capabilities for interacting with other devices. The modules can also be mechanically connected each other with magnet pairs and/or mechanical hinges placed on their external surface. The test prototypes do not include a power-pack and are powered externally using thin isolated wires. In the current design, the device 


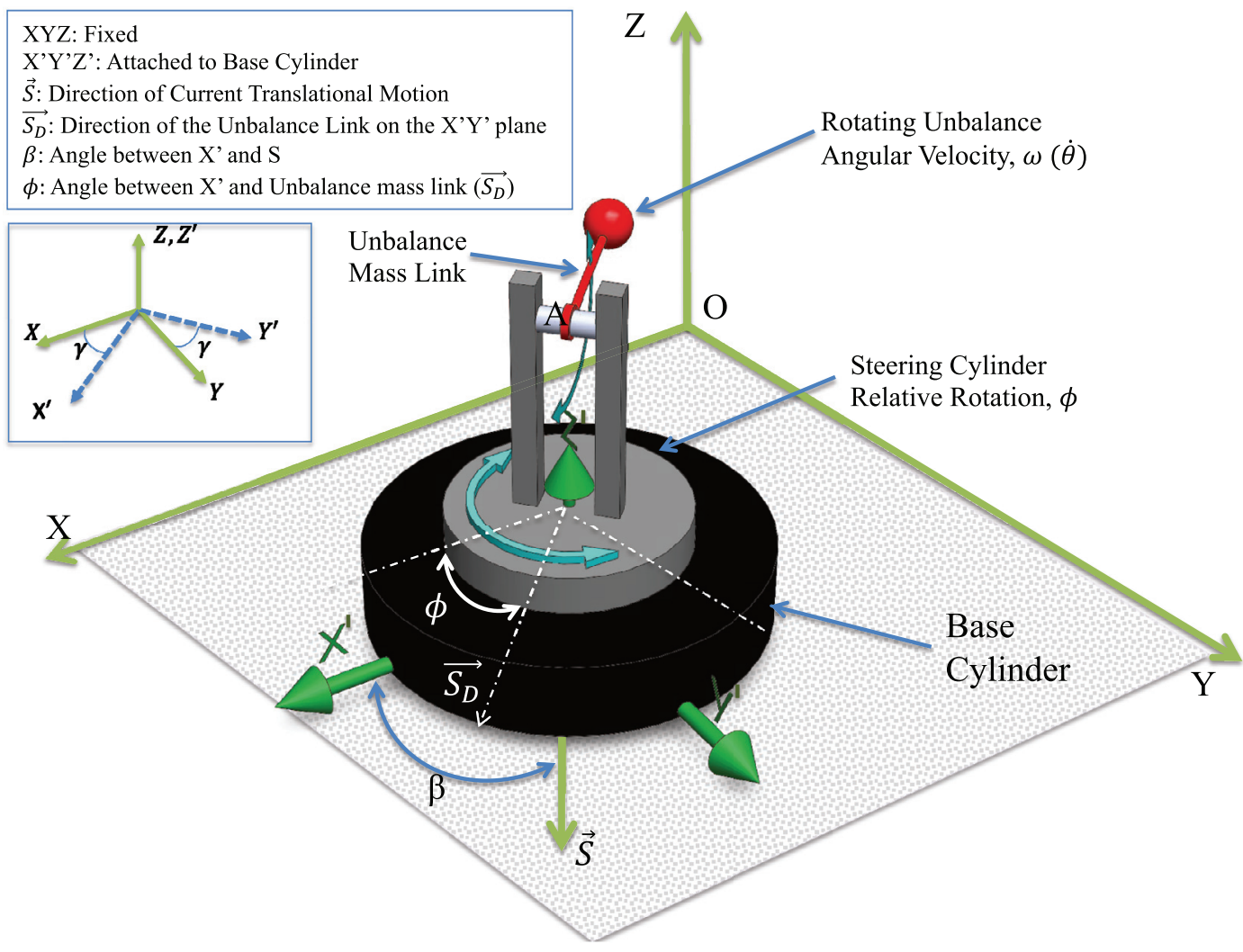

Fig. 3. The locomotion mechanism of the MechaCell using an unbalanced mass.

also have a secondary vibration motor attached at the top to control its orientation.

The integration and operation of sensing, actuation and computing hardware in the MechaCell system is explained in [14]. The mechanism that provides the locomotion of the module is a novel application of controlled unbalance motion as shown in Fig. 3. Rotational motion of the unbalance mass generates a force that causes the translational motion of the base cylinder. The mechanism consists of a rotating unbalance mass attached to a steering cylinder. The steering cylinder is attached to the base cylinder. For representing the motion of the body and the components, two coordinate systems are defined, one coordinate system (FCS) fixed at the origin, $O$ and a moving and rotating coordinate system (MCS) attached to the base cylinder such that the $Z^{\prime}$ axis is parallel to the $Z$ axis as shown in the figure. Steering-cylinder is attached to a base cylinder and can rotate about the $Z^{\prime}$ axis. The $S$ vector points to the current velocity of the MechaCell and makes an angle of $\beta$ radians with the $X^{\prime}$ axis. The unbalanced mass rotated by a DC-motor with an angular velocity of $\omega$ which is both perpendicular to the $Z^{\prime}$ axis and $S_{D}$, a vector points to direction of unbalanced mass link in the X'Y' plane. The steering cylinder is rotated with a servo motor housed in the base and its orientation relative with the $X^{\prime}$ axis is given as $\phi$. Although axes $Z$ and $Z^{\prime}$ are always parallel per the problem setup, the $X Y$ and $X^{\prime} Y^{\prime}$ pairs may have different orientations due to the rotation of the MechaCell during motion. An important parameter shown as a sidenote in Fig. 3 is the base cylinder rotation, $\gamma$, which is defined as the angle between the fixed axis $X$ and moving and rotating axis $X^{\prime}$.

As the un-balanced mass, $m$, rotates a planar reaction force, $\mathbf{F}_{u}^{\prime}$, is applied to the base from the link at connector A. The MechaCell frame moves depending on the current rotational speed of the unbalanced mass, the amount of the unbalance mass overcoming the forces due the friction, $\mathbf{F}_{f}$, between the body and the surface and the inertia. The application direction of the reaction force can be changed by rotating the steering cylinder (i.e. changing $\phi$ ) with respect to the base as shown in Fig. 3 using the attached servo motor.
In this paper, the precision planar motion control algorithm of a MechaCell is presented with both analysis (i.e. mathematical model and simulations) and experimental work. The proposed MechaCell system, which is designed as the building block element in these types of systems, have actuation capability with sensor and wireless connectivity in a closed package (i.e. no wheels and/or moving extensions). With the steady progress of electronics and sensor technologies, our primary motivation and focus is to design a reliable motion control system which is precise and suitable for using collaborative mechatronic applications such as positioning under strict constraints. The primary contributions of the research presented in this paper can be given as the mathematical model for a mechatronic device equipped with a novel locomotion system that includes friction and three dimensional centrifugal effects, a feedback linearizing control system method featuring an extended LuGre friction model for rapid directional changes validated with test results. The controlled co-operation of these devices using the design and algorithm presented here is the topic of an upcoming publication.

The outline of the remainder of the paper can be given as follows. In Section 2, a mathematical model that explains the planar motion of the device is developed. Then, in Section 3, the motion controller developed for the MechaCell device is presented. The experimental setup to validate the controller performance is given in Section 4. Finally, in Section 5, initial conclusions and future work will be discussed.

\section{Mathematical model}

In this section, a control oriented translational mathematical model for the MechaCell unit is developed. This model is used for initial simulations before experimental validation. Part of this model is also used as the estimation model in the friction compensation algorithm of the MechaCell's motion controller as explained in the next chapter.

The dynamic model for the motion of the MechaCell device can be developed using planar motion principles of a rigid body under the influence of a three-dimensional external force and a friction force due to 

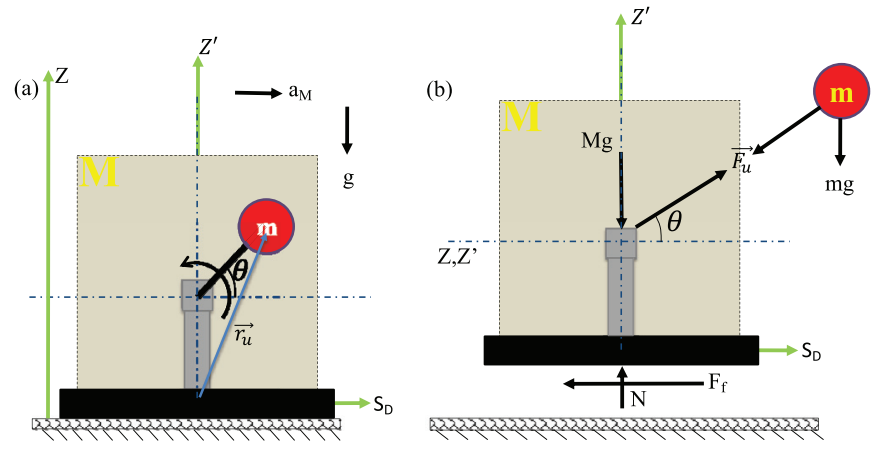

Fig. 4. Free body diagram of the system in Fig. 3.

sliding. One of the challenges associated with the system setup is that the motion force is generated by the acceleration of a component (unbalanced mass) attached to a base frame that does planar translation and rotation motion. Therefore, a relative velocity and acceleration analysis using the fixed (FCS) and moving (MCS) coordinate systems is more appropriate to find the correct locomotion effect of the unbalance mass.

In Fig. 4, forces affecting the motion of the MechaCell is shown using a projected view from $Z^{\prime} S_{D}$ plane given in Fig. 3 . The base can translate in $X$ and $Y$ axis directions. In addition, the unbalanced mass can rotate about the $Z^{\prime}$ axis and about the axis both perpendicular to $S_{D}$ and $Z^{\prime}$. Because of its motion it can be assumed that the unbalanced mass, $m$, has displacement, velocity and acceleration in three dimensions. The acceleration of the unbalanced mass, $\mathbf{a}_{\mathbf{m}}$, can be represented in components by using the orthogonal unit vectors $\mathbf{i}, \mathbf{j}$ and $\mathbf{k}$ in $X, Y$ and $Z$ directions respectively as shown in (1).

$\mathbf{a}_{\mathbf{m}}=a_{m, x} \mathbf{i}+a_{m, y} \mathbf{j}+a_{m, z} \mathbf{k}$

From Fig. 4 , the force, $\mathbf{F}_{\mathbf{u}}$, exerted by the rotating unbalanced mass on the MechaCell, can be calculated using (2).

$\mathbf{F}_{\mathbf{u}}=m \mathbf{a}_{\mathbf{m}}+m g \mathbf{k}$

As (2) implies, calculation of unbalanced force, $\mathbf{F}_{u}$, requires the calculation of the inertial acceleration of the unbalanced mass, $m$. In
Fig. 5(a) the components of the acceleration of the unbalanced mass is shown for a simple linear motion case. It can be seen clearly that the acceleration, $\mathbf{a}_{M}$, of the base cylinder is also a contributor to the inertial acceleration of the unbalanced mass. It is also important to note that additional terms may be contributors since the rotation of the base cylinder and the steering mechanism also can cause rotation to the unbalanced mass as shown in Fig. 3. The total acceleration, $\mathbf{a}_{m}$, of the unbalanced mass of the system can be calculated by using the relative acceleration equation given in (3) by assuming $X Y Z$ as the fixed axis (FCS) and $X^{\prime} Y^{\prime} Z^{\prime}$ as the moving axis (MCS) coordinate systems.

$\mathbf{a}_{\mathbf{m}}=\mathbf{a}_{\mathbf{M}}+\ddot{\gamma} \times \mathbf{r}_{\mathbf{u}}+\mathbf{a}_{\mathbf{r e l}}+\dot{\gamma} \times\left(\dot{\gamma} \times \mathbf{r}_{\mathbf{u}}\right)+2\left(\dot{\gamma} \times \mathbf{v}_{\text {rel }}\right)$

In (3), $\mathbf{a}_{\mathbf{M}}, \dot{\gamma}$ and $\ddot{\gamma}$ are the acceleration, angular velocity and angular acceleration of the MechaCell base (i.e. the moving frame) respectively. $\mathbf{r}_{\mathbf{u}}, \mathbf{v}_{\text {rel }}$ and $\mathbf{a}_{\text {rel }}$ are the position, velocity and acceleration of the unbalanced mass with respect to center of the base component. In Fig. 5(b) the effect of omission of the base acceleration and rotational components and using only $\mathbf{a}_{r e l}$ is shown. Since the omission overestimates the locomotion force, a $0.3 \mathrm{~cm}$ steady state error is observed for a single pulse motor command (given at $100 \mathrm{PWM}$ (40\% duty cycle) level for $4 \mathrm{~s}$ ) simulation. Using $\mathbf{a}_{r e l}$ only for unbalanced force estimation is common practice. However, this difference is important to note for applications where precise movement is a requirement. Therefore the moving frame acceleration calculation given in (3) will be used in the controller design algorithms and system simulations.

As shown in Fig. 4, forces acting on the MechaCell system are the weight and normal forces in the $Z^{\prime}$ direction, friction force, $\mathbf{F}_{f}$, in the opposite of the velocity direction, $\mathbf{S}$, and reaction force, $\mathbf{F}_{\mathbf{u}}$ the rotating in the $Z^{\prime} S_{D}$ plane. Applying Newton's Second Law to the MechaCell with mass, $M$, we can obtain the dynamic motion equations as given in (4)(6).

$$
-M g+F_{u} \sin (\theta)+N=M a_{M, z}=0
$$

$F_{u} \cos (\theta) \cos (\phi+\gamma)-F_{f} \cos (\beta+\gamma)=M a_{M, x}$

$F_{u} \cos (\theta) \sin (\phi+\gamma)-F_{f} \cos (\beta+\gamma)=M a_{M, y}$
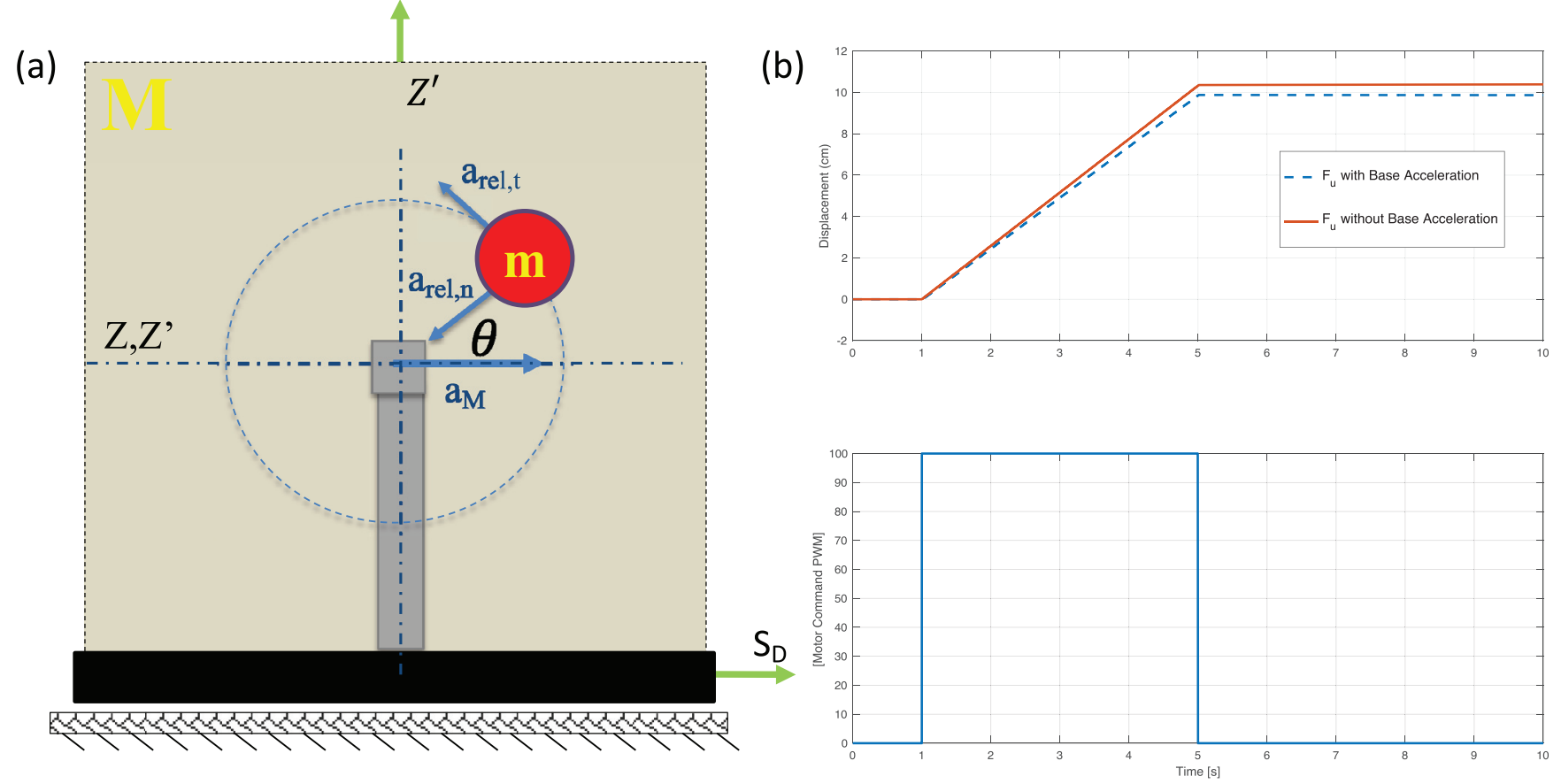

Fig. 5. Acceleration of the Unbalanced Mass (a)Base and Relative Acceleration (b) The Effect of base acceleration in $F_{u}$ calculations. 
Table 1

Parameters used in the mathematical model.

\begin{tabular}{lll}
\hline Mass of the Mechacell & $M$ & $0.049[\mathrm{~kg}]$ \\
Mass of the rotating unb. & $m$ & $0.006[\mathrm{~kg}]$ \\
Inertia of the Mechacell & $I$ & $0.0000123\left[\mathrm{kgm}^{2}\right]$ \\
Radius of the Mechacell & $r_{M}$ & $0.025[\mathrm{~m}]$ \\
Radius of the rot. unb. & $r$ & $0.004[\mathrm{~m}]$ \\
Angular speed of the unbalance & $\omega$ & Swept $[\mathrm{rad} / \mathrm{s}]$ \\
Coefficient of static friction & $\mu_{s}$ & 0.2 \\
Coefficient of kinetic friction & $\mu_{k}$ & 0.15 \\
Force prod. by the unbalance & $F_{u}$ & Calculated $[\mathrm{N}]$ \\
Torque prod. by the sec. motor & $T_{s}$ & Calculated $[\mathrm{Nm}]$ \\
Friction force & $F_{f}$ & Calculated $[\mathrm{N}]$ \\
Friction torque & $T_{f}$ & Calculated $[\mathrm{Nm}]$ \\
Normal force & $N$ & Calculated $[\mathrm{N}]$ \\
Gravitational constant & $g$ & $9.81\left[\mathrm{~m} / \mathrm{s}^{2}\right]$ \\
\hline
\end{tabular}

where $N$ is the normal force applied on the base from the surface and $F_{f}$ is the magnitude of the friction force experienced by the base in the direction opposite to the motion. The rotational dynamics of the MechaCell structure by using the derivative of the total angular momentum about its center of mass as shown in (7).

$I \ddot{\gamma}=T_{s}+T_{u}-T_{f}$

In (7), $T_{s}$ is the torque provided by the secondary motor, $T_{u}$ is the reaction torque generated by the unbalanced mass motion on the MechaCell structure. Since it involves higher order sinusoidals multiplied with small moment arm values, the contribution of $T_{u}$ is very small and currently disregarded. As an example, the torque generated by the unbalanced mass can be calculated by using the physical parameters. At the maximum condition when $\theta=0$, the torque can be estimated as $T_{u}=\operatorname{rmr}(\ddot{\gamma}+\ddot{\phi})$, considering the rotational motion of unbalanced mass, $m$ as viewed from the $X^{\prime} Y^{\prime}$ in Fig. 3. The steering cylinder has a maximum rotational acceleration of $\ddot{\phi}=500 \mathrm{rad} / \mathrm{s}^{2}$ (device specifications) and the base cylinder rotation rate is less than approximately $1000 \mathrm{rad} / \mathrm{s}^{2}$ based on experimental data, this gives us a unbalanced torque value of $T_{u} \approx 8 \times 10^{-5} \mathrm{Nm}$ as an estimated maximum value using the values given in Table 1. In (7), $I$ and $\gamma$ are the rotational inertia and rotation of the MechaCell about $Z^{\prime}$ axis respectively. $T_{f}$ is the friction torque generated by the friction force between the plane surface and the MechaCell floor and can be approximated by $T_{f}=r_{M} F_{f}$ (friction force applied at the rim) where $r_{M}$ is the radius of the MechaCell device using the single friction clutch plate calculations given in references such as [16]. Likewise, we can estimate the static friction torque, $T_{f, s}$ by using the formula $T_{f, s}=r_{M}(M+m) g \mu_{s} \approx 1 \times 10^{-2} \mathrm{Nm}$. $T_{s}$ varies on command, however, comparison of the static friction torque and the estimated unbalance torque values supports the decision to neglect $T_{u}$, in (7).

For precise control of the MechaCell motion the calculation of the friction force is crucial. The friction force between the surface and the lower surface of the MechaCell base can be estimated using various models. For example, using the Coulomb Friction Model, the friction force can be calculated by using the normal force, $N$, acting on the MechaCell pads and a friction coefficient [17]. A Coulomb friction force estimation, $F_{c}$ can be calculated as shown in (8).

$\mathbf{F}_{\mathbf{c}}=-\mu_{k} N \mathbf{e}_{\mathbf{v}}=-\mu_{k}\left(M g-F_{u} \sin (\theta)\right) \mathbf{e}_{\mathbf{v}}$

where $\theta$ is the current orientation of the unbalanced mass link, $\mathbf{e}_{\mathbf{v}}$ is a unit vector in the direction of the velocity of the MechaCell and its in the direction of the translation vector $\mathbf{S}$. $\mu_{k}$ is the friction coefficient when the device is in motion. The calculation of the friction force with the Coulomb approach results in a simple and quick estimation. However, the friction force is known to change with various factors as noted by many researchers and more detailed models exist [18-21] including the flexibility of the contact interactions and direction. One of these mod- els is known as the LuGre model and can be represented as shown in (9)-(12).

$$
\begin{aligned}
& F_{f}=\sigma_{0} z+\sigma_{1} \dot{z}+f(v) \\
& \dot{z}=v-\sigma_{0}|v| / g(v) z \\
& g(v)=F_{c}+\left(F_{s}-F_{c}\right) e^{-\left|v / v_{s}\right|^{\alpha}} \\
& f(v)=\sigma_{2} v
\end{aligned}
$$

The friction model given between (9) and (14) have many application specific parameters. $F_{s}$, represent the stiction force and is calculated using $F_{s}=\mu_{s} N . f(v)$ is the viscous friction force therefore $\sigma_{2}$ is the viscous friction coefficient which determined to be around $0.3 \mathrm{Ns} / \mathrm{m} . \alpha$ in function $g(v)$ is taken as 1 based on the discussion given in [22]. $z$ is an internal state of the friction model and spring and damper constants, $\sigma_{0}$ and $\sigma_{1}$ are taken as $13 \mathrm{~N} / \mathrm{mm}$ and $6 \mathrm{Ns} / \mathrm{mm}$ respectively. $v_{s}$ is the exponential decay constant in function $g(v)$ and a value of $8 \mathrm{~mm} / \mathrm{s}$ is used. Although physical interpretations exists, in order to determine these values, the estimated value of the friction force, $F_{f}$, was determined from the experimental data points using $F_{f}=F_{u, \max }-(M+m) a_{M}$ and recorded with the corresponding velocity. Since at each data point motor command is known, but the angular position of the unbalanced mass, $\theta$, is not measured, a maximum unbalance torque $F_{u, \max }$ was calculated using the motor command data. Then, using the calculated $F_{f}$ vs. $v$ pairs based on the experimental data points given in Fig. 6(a), fitting LuGre model parameters were calculated. The model fit is given in the same figure as a solid line.

In the original form of the LuGre model, friction force generated due to the translation motion in the direction of motion is considered. However, in our system there is also rapid directional change of motion that affect the friction force experienced by the MechaCell due to rapid rotation of the steering base (i.e. rapid change of $\phi$ ). Therefore, a second stable state, $\xi$ was added that becomes active as the device changes $F_{u}$ direction on the $X Y$ plane and quickly converges to zero if the new direction is conserved as shown in (13). In calculations, to obtain a reasonable delay, $\sigma_{3}$ is used as $0.04, h$ function was set to $\pi|\dot{\phi}|$ and $\sigma_{4}=0.05 \mathrm{~N} / \mathrm{rad}$ was used. The calculated effect of the rotational angle extension to the LuGre friction model is presented in Fig. 6(b). The data points show the calculated value of friction, $F_{f}$, when the device have a velocity between 0.005 and $0.05 \mathrm{~m} / \mathrm{s}$ range with changing steering cylinder velocity, $\phi$. The effect of friction is seemingly high in the low $\dot{\phi}$ values since this is also the high acceleration start area.

The parametes in the LuGre extension given in (13) and (14) are chosen accordingly as presented in Fig. 6(b).

$\dot{\xi}=\dot{\phi}-\sigma_{3}|\dot{\phi}| / h(\dot{\phi}) \xi$

Then the total friction calculation given in (9) can be modified as shown in (14). The direction of the friction force is the opposite of motion, i.e. in the $-\mathbf{e}_{\mathbf{v}}$ direction.

$F_{f}=\sigma_{0} z+\sigma_{1} \dot{z}+f(v)+\sigma_{4} \xi$

The force applied by the unbalanced mass to the system is a function of the motor rotational speed $\omega$ since the unbalanced mass and the link length do not change. The motor rotational speed is a function of the command signal which is given as a Pulse Width Modulation (PWM) signal. PWM signal is a standard input signal used in power electronics and its typical designator is the width modulation [23] given in the units of duty cycle percentage $\% D C$. The constant that relates the PWM signal to the maximum unbalance force, $F_{u}$ is calculated experimentally as $K_{v b}=13 \times 10^{-6}$ where $F_{u}=K_{v b} \times \% D C \mathrm{mN}$. In Table 1 , all the major parameters with numerical values (if applicable) used in the simulation of the mathematical model are summarized.

In Fig. 7, comparison between the mathematical model and experimental data from the real system is shown for a single direction 


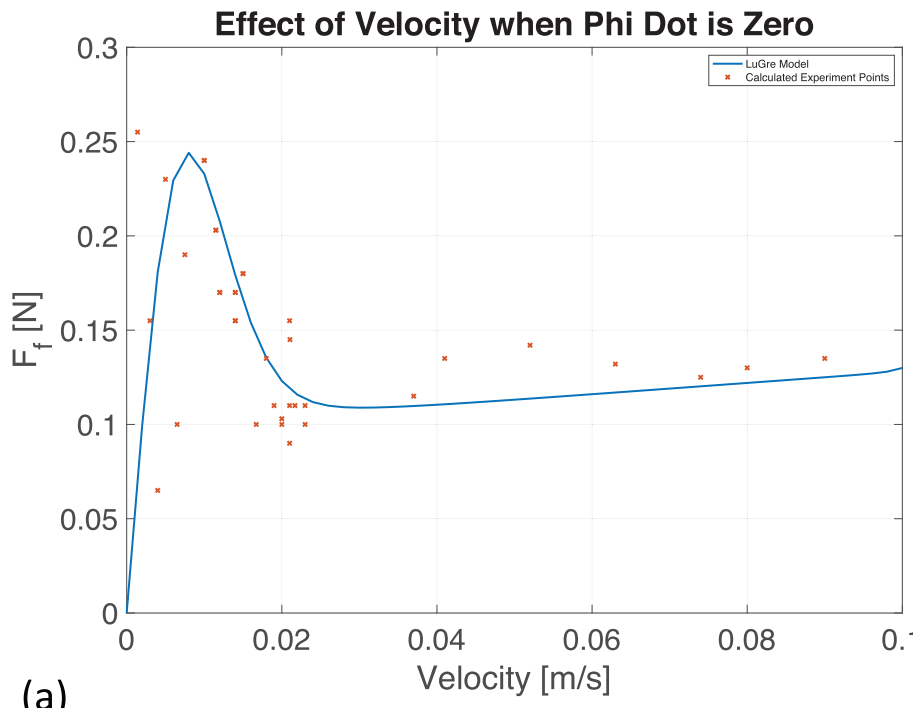

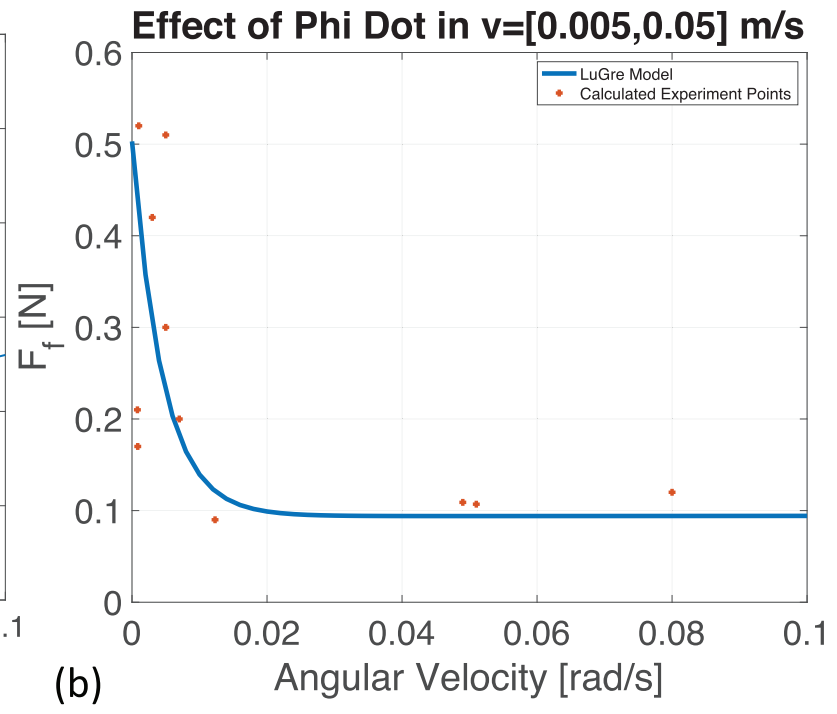

(b)

Fig. 6. (a) Identifying LuGre Model Parameters (b) The effect of base speed on friction calculations.

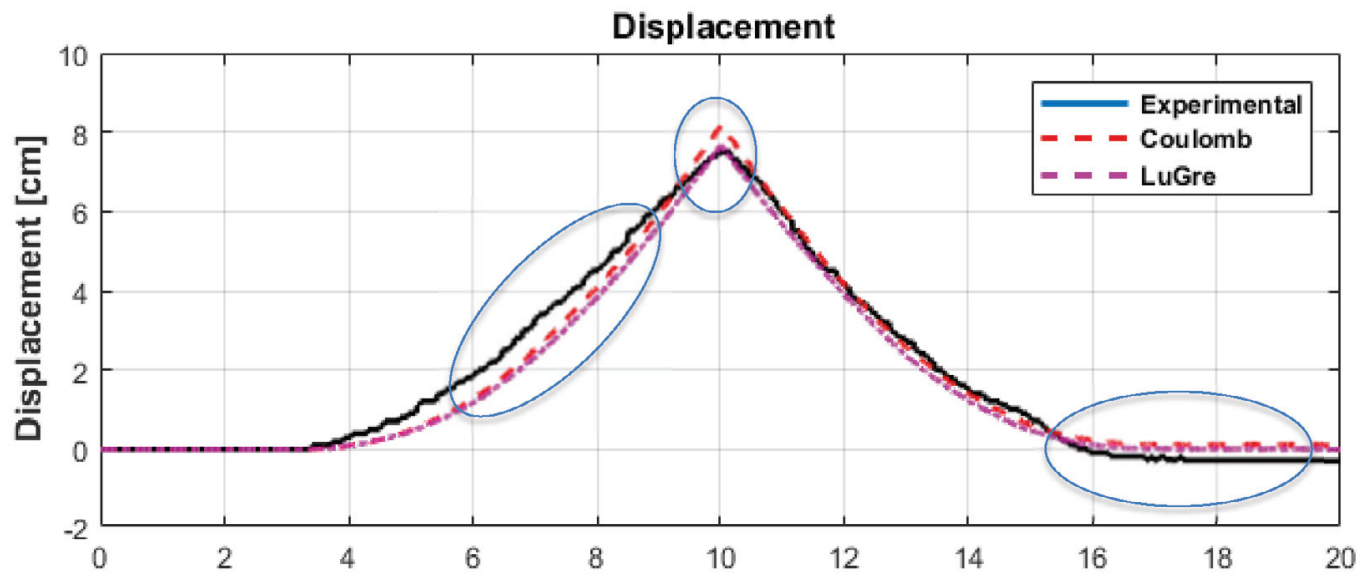

PWM signal

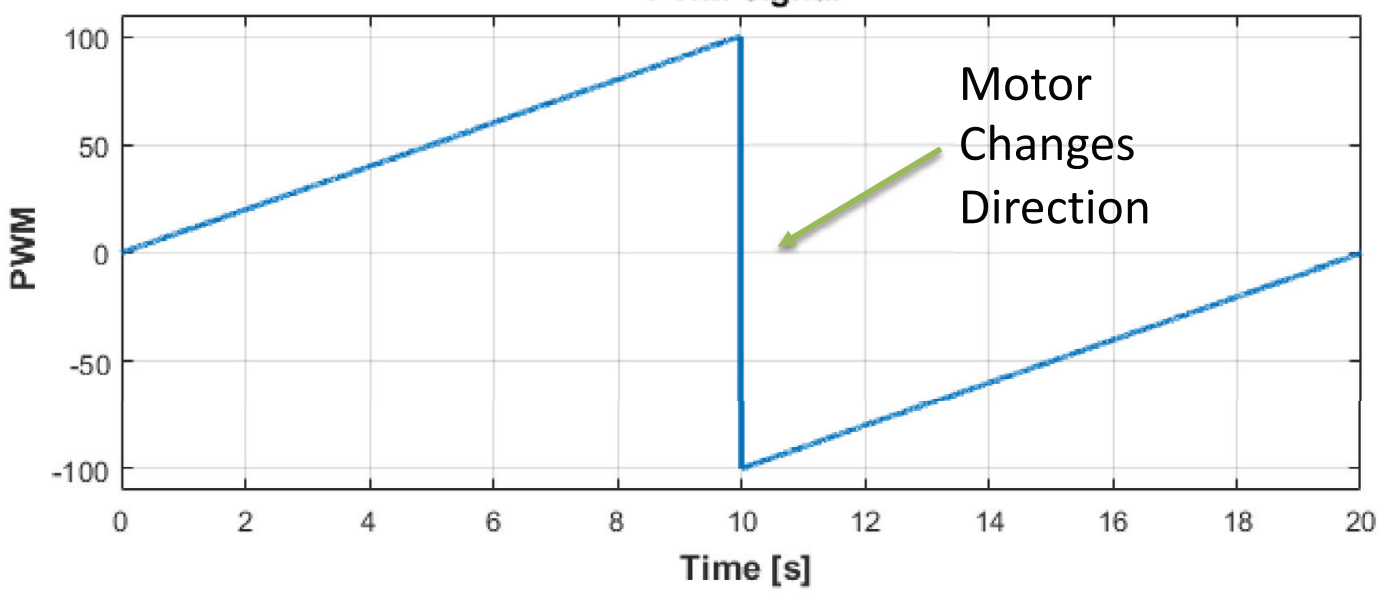

Fig. 7. Comparison of the dynamic models with different friction calculations. 


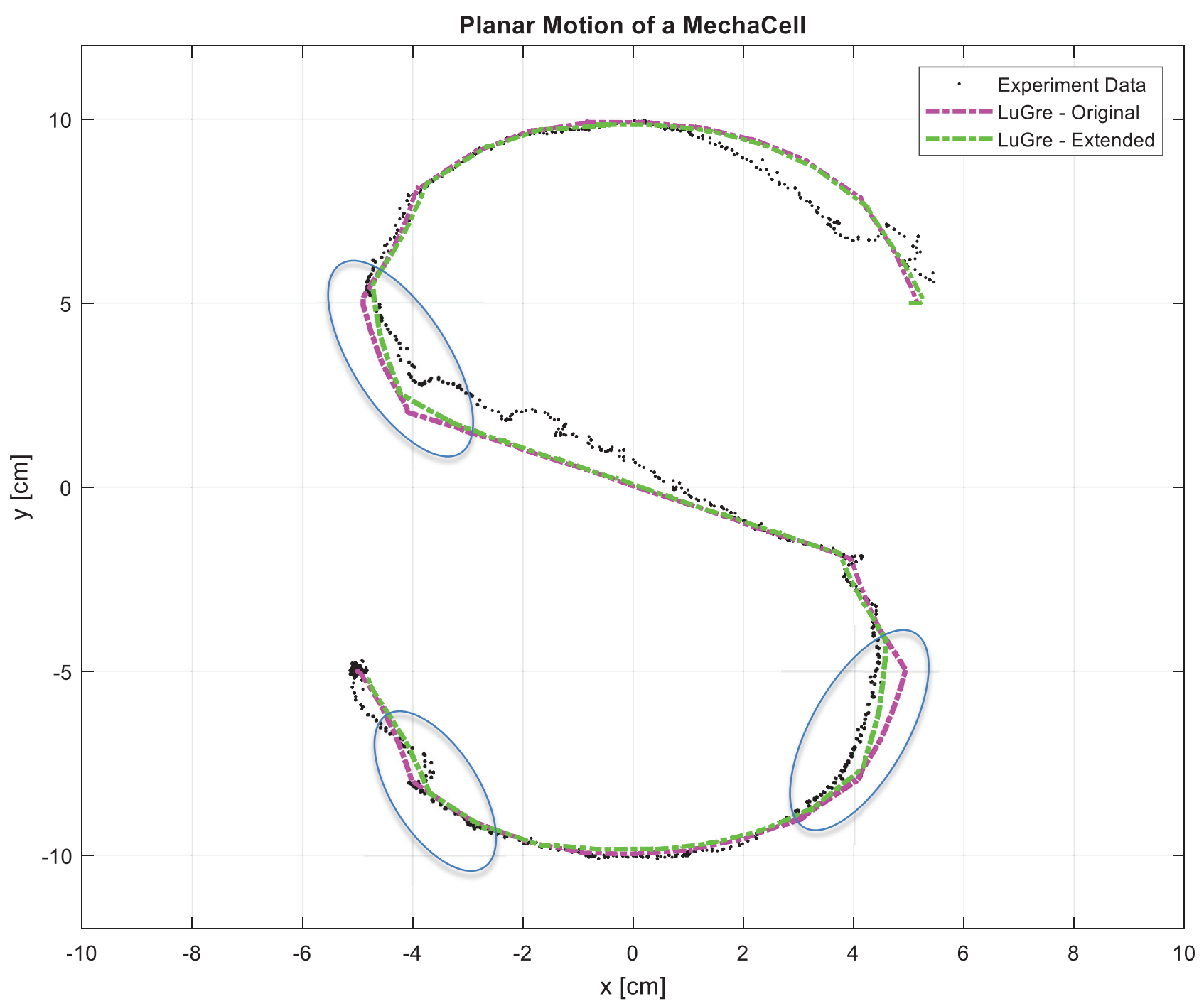

Fig. 8. Comparison of the mathematical models in planar motion with the experimental data.

translation (rectilinear motion) case. The speed command value is increased from 0 to 100 in $10 \mathrm{~s}$, then the polarity is reversed (i.e. direction of rotation is changed) and the command is decreased from 100 to 0 with constant slope. The calibration for motor command and angular speed is given as $14.65 \mathrm{rad} / \mathrm{s} / \% \mathrm{DC}$. It can be seen that the model using LuGre friction model, which includes the velocity and flexible interactions (effective in the encircled regions) and the effect of base acceleration of the contacting surfaces is predicting the real world response better than conventional Couloumb friction model which only includes the normal force. In this case, since the steering wheel is not moving, there is no change in the direction of MechaCell and the state, $\xi$, is always 0 and therefore not contributing to the friction calculations. The friction identification experiments also show a delayed start that points to the existence of motor driver circuit and communication related delays. This delay is not studied in this work. In Fig. 8, instead of single direction motion, a planar translation profile was used where the steering wheel rotates during the motion as well. It can be seen from the results that modification on the LuGre model, which compensates for the direction changes of the sliding surface, predicts the friction force better for the planar motion. The encircled regions in the figure show this compensation in accord to the experiment data when the steering system of the MechaCell device is used. Better match with experimental data is possible, however, only the correct direction of compensation is targetted to avoid over-calibrating.

\section{Motion controller}

In Fig. 9, the overview of the closed-loop control system used in the motion control of the MechaCell device is presented. The desired planar position and orientation is communicated to the device using wireless messaging. Since the MechaCell moves using the unbalanced mass

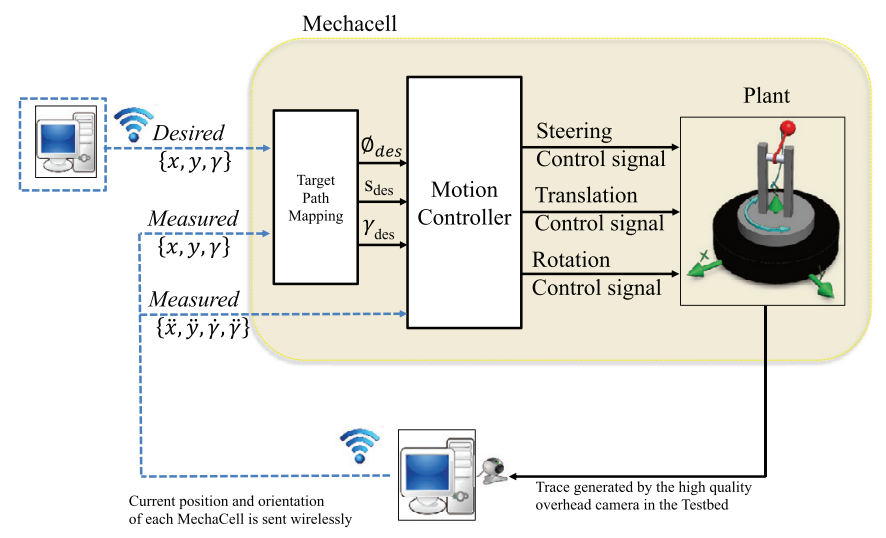

Fig. 9. Position feedback control scheme of the MechaCell. 
reaction force that generates motion in one direction, the desired position values are converted to desired translation and direction information using the "Target Path Mapping" calculations. The testbed used in this study have the high definition image capture capability and measured acceleration is sent to each device as a wireless message. All of this information is then used by the "Motion Controller" to generate the translation and rotation control signal sent to the MechaCell motors. In the remainder of this section target path mapping and motion controller features shown in this figure will be discussed.

In Fig. 10, desired translation and direction generation based on the desired Cartesian coordinates of the MechaCell device is presented. At a typical instant MechaCell should travel from its current position, $\mathbf{R}_{\mathbf{M}}$, following the vector starting at its current position and ending at the desired position, $\mathbf{R}_{\mathbf{D}}$. This vector is shown as $\mathbf{S}_{\mathbf{D}}$. Hence, to move towards the desired point, the servo controller should position the steering cylinder such that $\angle \mathbf{S}_{\mathbf{D}}=\phi+\gamma$ where $\gamma$ is the orientation of the MechaCell with respect to the fixed frame $X Y$ and $\phi$ is the angle of the unbalanced mass link with respect to the moving and rotating frame $X^{\prime} Y^{\prime}$.

The desired translation reference used by the s-controller, and the servo controller is shown in (15).

$s_{\text {des }}=\left|\mathbf{R}_{\mathbf{D}}-\mathbf{R}_{\mathbf{M}}\right|$

where, $\mathbf{R}_{\mathbf{D}}$ and $\mathbf{R}_{\mathbf{M}}$ are the desired and MechaCell's current position vectors w.r.t. the inertial coordinate system. The desired direction of the translation can be calculated by using the direction of the $\mathbf{s}_{\text {des }}$ and current orientation of the Mechacell on the XY plane, $\theta$ which is a sensor input as shown in (16).

$\phi_{\text {des }}=\angle \mathbf{S}_{\mathbf{D}}-\gamma$

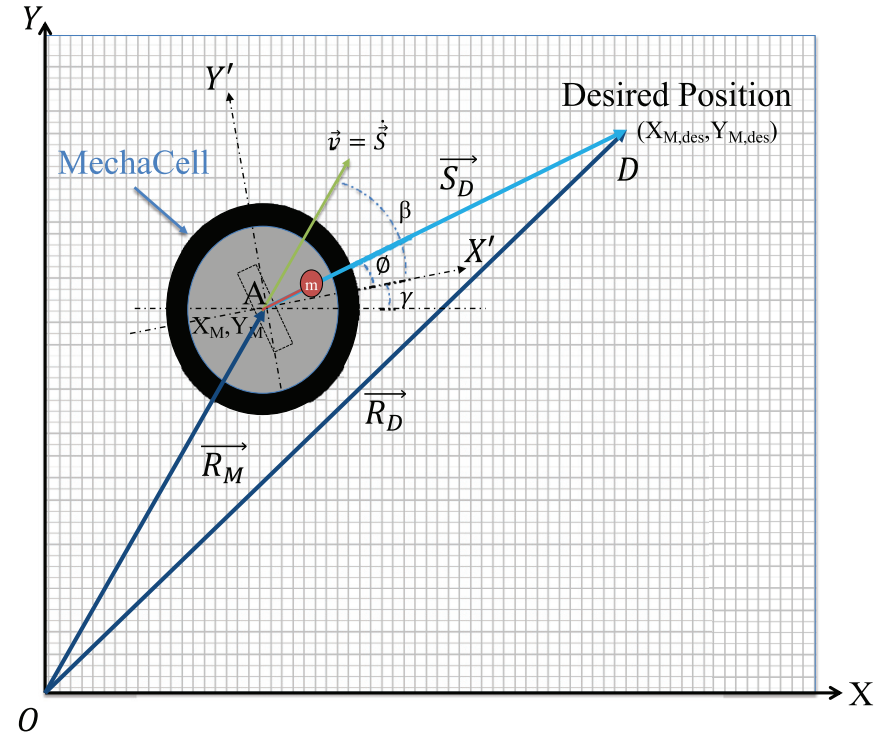

Fig. 10. Top view of the MechaCell, its position vector, position vector of the desired position, MechaCell's orientation and steering-cylinder orientation.

During the simulations and experiments performed for this work, the desired orientation of the MechaCell, $\gamma_{\text {des }}$, is commanded as $0^{\circ}$ so that the fixed and moving frames are kept aligned. In other cases, depending on the application, the angular orientation can also be controlled as a function of time using the reference input.

In Fig. 11, interaction of the "Motion Controller" and "Plant" blocks given in Fig. 9 are explained in detail. The motion controller has

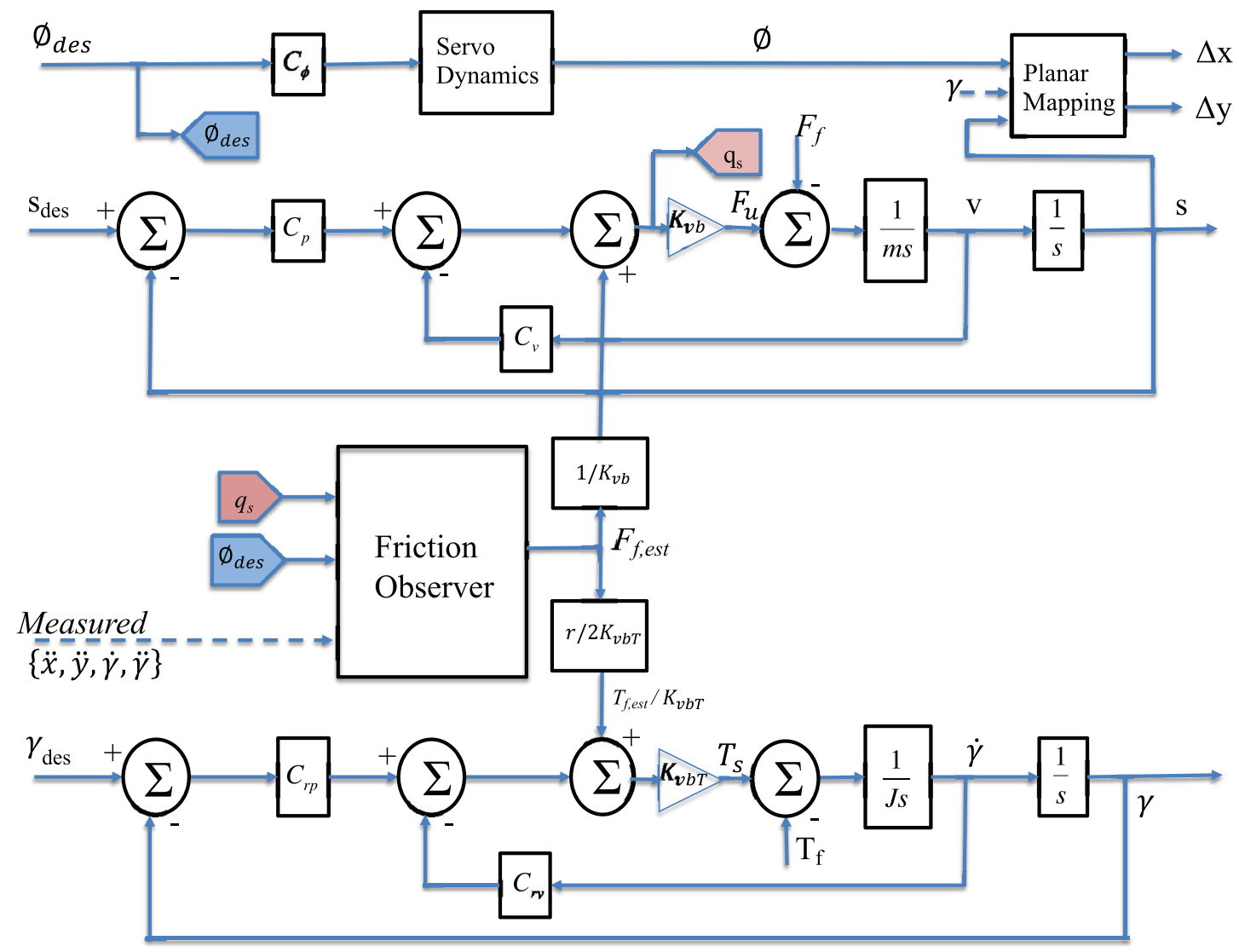

Fig. 11. The Motion Control algorithm for the MechaCell Device. 

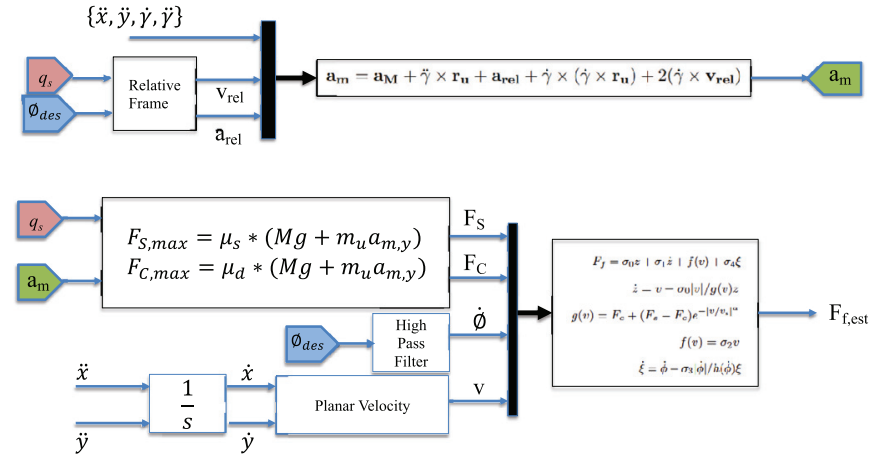

Fig. 12. Friction Observer (from Fig. 11) with heading and inertial compensation.

three subsystems: an open loop steering servo command function that turns the steering cylinder to the desired angle, a translation controller (s-controller) controls the motion of the MechaCell at the current heading by controlling magnitude to the actuating force through rotational velocity of the unbalanced mass, and an orientation controller ( $\gamma$-controller)-controlling orientation of the MechaCell device by commanding a reaction torque generated by the second vibration motor attached at the top of the MechaCell.

The control algorithm also features an observer that predicts the friction force experienced by the device and the commanded unbalanced mass rotational speed is compensated accordingly. The friction observer block shown in Fig. 11 is given in detail in Fig. 12. The basis for the friction compensator is the friction model explained in the previous section based on the underlying equations given in (1)-(14). These set of nonlinear equations receive the current translational acceleration, current controller command, $q_{s}$, servo command, $\phi_{\text {des }}$, device translational acceleration components, $\{\ddot{x}, \ddot{y}\}$, rotational velocity and acceleration (i.e. $\dot{\gamma}$ and $\ddot{\gamma}$ ) as the input and calculate the estimated friction force acting on the system. In the implementation of the friction estimator, first the acceleration of the unbalanced mass, $a_{m}$ can be calculated using the sensor data and the controller signals, then a maximum value for the unbalanced force, $F_{u}$ can be found using the controller command by also including the current acceleration of the unbalanced mass. This value and the velocity information can be used in Eqs. (8) and (9) to estimate the current friction force $F_{f}$ experienced on the base surface. The stability of the LuGre friction model algorithm was studied by many researchers $[18,24]$. It can also be seen that the extended model used in this paper is also stable since the added state, $\xi$, given in Eq. (12) is stable and decoupled from the rest of the plant dynamics.

When the friction estimation algorithm is implemented with properly calibrated parameters, the estimation error, $e_{F, f}=F_{f, e s t}-F_{f}$ is small, the contribution from the friction force can be assumed as a disturbance and the controllers can be designed accordingly as a second order system with inertia dynamics. In fact, with $e_{f} \approx 0$ the transfer functions representing the translation dynamics (i.e. $G_{s}(s)=S(s) / S_{\text {des }}(s)$ ) and the rotation dynamics (i.e. $\left.G_{\gamma}(s)=\gamma(s) / \gamma_{\text {des }}(s)\right)$ in Fig. 11 can be given as shown in (17) and (18) respectively.

$G_{s}(s)=\frac{C_{p} K_{v b}}{m s^{2}+C_{v} K_{v b} s+C_{p} K_{v b}}$

$G_{\gamma}(s)=\frac{C_{r p} K_{v b T}}{I s^{2}+C_{r v} K_{v b T} s+C_{r p} K_{v b T}}$

where $C_{p}, C_{v}$ velocity and position controller parameters for the translation loop and $C_{r p}, C_{r v}$ are the rotational position and velocity controller parameters for the rotation loop respectively. Constants $K_{v b}$ and $K_{v b T}$ are signal-to-effort servomotor constants as discussed before. Based on the inertia properties of the MechaCell devices these unity gain transfer functions are tuned to have critically damped nature so that the response obtained will have no overshoot but fast response. The value selected for $C_{p}$ also affects the noise rejection property of the system. The $C_{p}$ and $C_{v}$ values selected for the s-control loop are 10 and 29 respectively. For the $\gamma$-control loop, 0.1 and 180 are used for $C_{r v}$ and $C_{r p}$ parameters respectively.

\section{Simulation and experiments}

After the mathematical model and the motion controller are prepared as explained in the previous sections, an experimental setup was constructed for validation. The experimental setup in the laboratory consists of five major components: a PC with Bluetooth capability, a table top platform, an overhead camera, a power-source, MechaCells and a workpiece as shown in Fig. 13.

Device tracking algorithm is based on color recognition and the information is then send to the MechaCells through Bluetooth. Experiments are performed on a platform over which the overhead camera is placed for motion tracking. The platform is $50 \times 50 \mathrm{~cm}$ and its floor is made of Styrofoam. MechaCells are powered with an external power-source of $10[\mathrm{~V}]$. To eliminate the influence of a tether on MechaCell's motion a very thin wire (magnetic wire) is used.

Using the constructed experimental setup, single MechaCell path following experiments are performed to see the effectiveness of the control algorithm developed. For the real experiments same controller calibration is used as in the Matlab/Simulink simulations as explained in the previous section and Figs. 9 and 11. Path tracking simulation and experiment results are presented in Fig. 14. In Table 2 as numerical error parameters, deviation from desired position in $x$ and $y, x_{e r r}$, and, $y_{e r r}$, respectively and deviation from path, $\epsilon$, from the experiment and simulations are listed.

As it can be seen from Fig. 14, simulation results are much better than real-life experiments because of the noise and disturbance in the real setup most importantly coming from the overhead tracking camera. Starting points of all data is normalized for $(-9 \mathrm{~cm},-10 \mathrm{~cm})$ point for fair comparison. However, when the experiments are compared to each other, the extended LuGre compensation which includes the effect

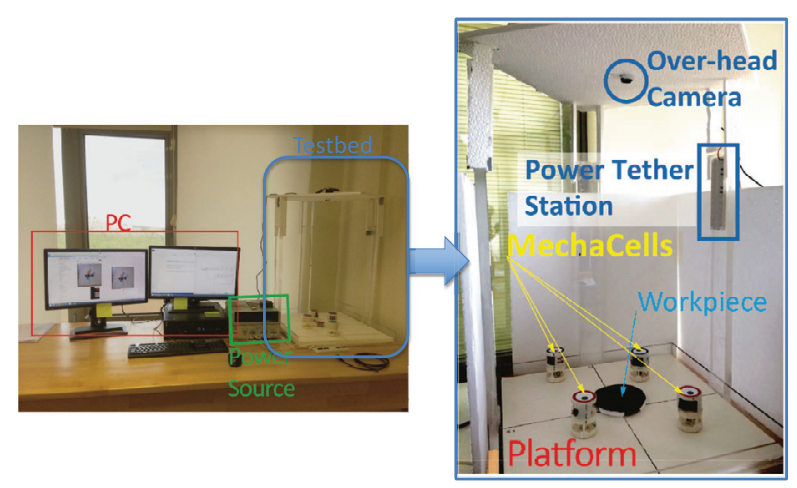

Fig. 13. Experimental setup in the laboratory.

Table 2

Numerical values of the error parameters from the experiment and the simulations.

\begin{tabular}{|c|c|c|}
\hline$x_{e r r}[m]$ & $y_{e r r}[m]$ & $\epsilon[m]$ \\
\hline \multicolumn{3}{|c|}{ Simulation: Extended Lugre } \\
\hline 0.00030 & 0.00035 & 0.00015 \\
\hline \multicolumn{3}{|c|}{ Experiment 1: Original LuGre } \\
\hline 0.00490 & 0.00360 & 0.00140 \\
\hline \multicolumn{3}{|c|}{ Experiment 2: Extended LuGre } \\
\hline 0.00420 & 0.00290 & 0.00100 \\
\hline \multicolumn{3}{|c|}{ Experiment from [14] : PID+Coulomb } \\
\hline 0.00470 & 0.00330 & 0.00120 \\
\hline
\end{tabular}




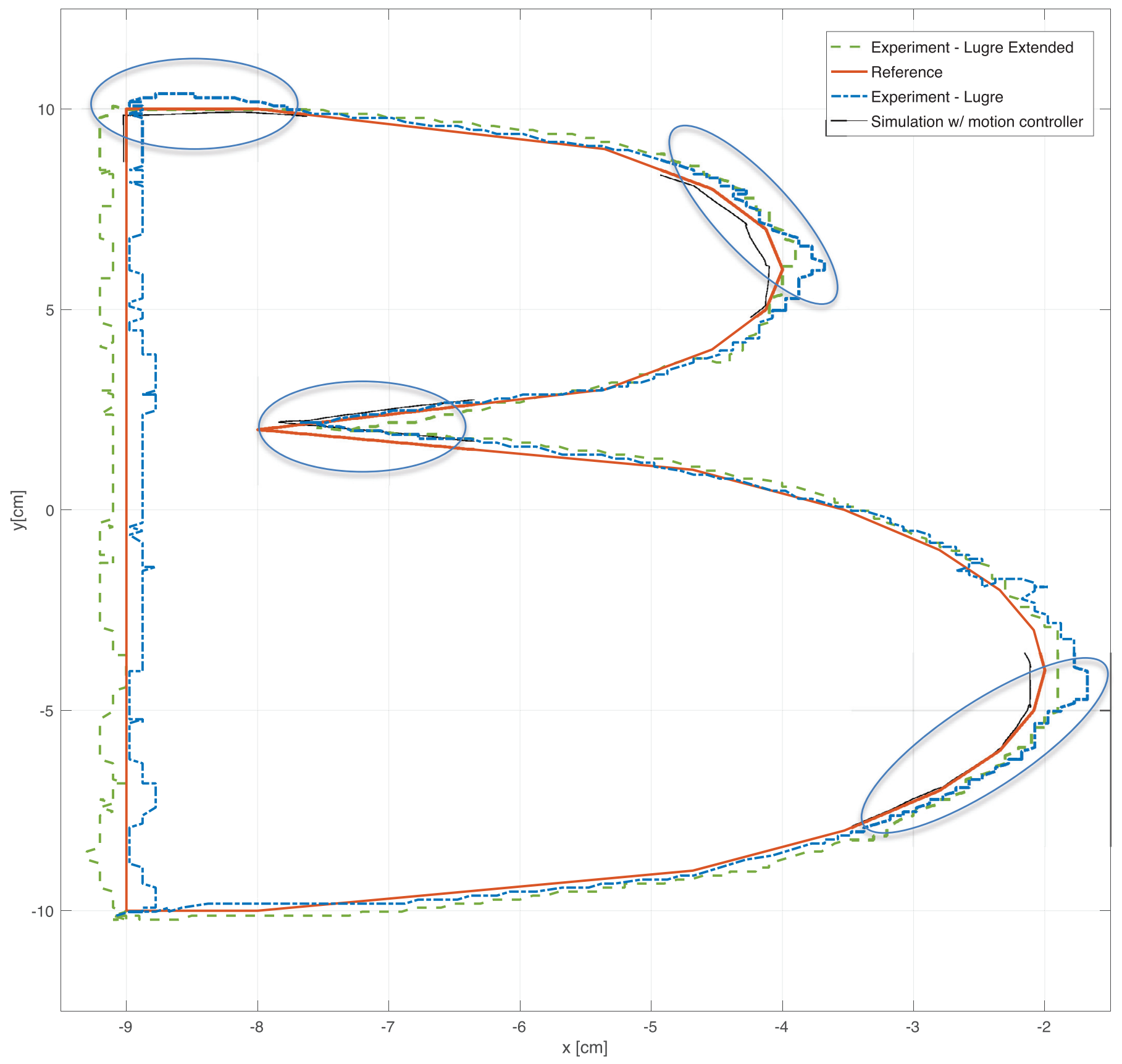

Fig. 14. Results of the path following tasks of a single MechaCell with experiments and simulation.

of rapid direction change of the MechaCell tracks the path better in during sharp turns. When the direction change is slow, both algorithms behave the similar as expected. The tracking results are also tabulated in Table 2. The extended LuGre compensator error results are better as the system performs better in sharp turns of the "B" shape, and overall $14 \%$ improvement in the contour error. The extended LuGre algorithm also performed better than the highly calibrated PID controller used in [14].

Our experiments and calculated error data show that a successful motion controller is developed for the MechaCell system including a direction aware LuGre friction compensation algorithm. This controller gives better tracking results than using a conventional friction compensator only. In Fig. 15, the motor control command and the friction compensation is given for the B-shape experiment presented in Fig. 14 "Extended LuGre" version. In order to show the effect of the directional compensation the important parts that corresponds to different parts of the travel are highlighted in boxes with numbers. In dashed box numbered 1, the device makes its first sharp turn and the compensation reacts to that with increased command even though the speed (slope of the first plot) did not change considerably. In dashed box 2, this is repeated for the next sharp turn in the B-shape. The reason for the dual correction is the fact that our steering motor only rotates between $0^{\circ}$ and $180^{\circ}$ and changes rotation direction for angles between $180^{\circ}$ and $360^{\circ}$ as explained in [14]. Therefore, it negotiates this sharp turn in two commands as shown in the second subplot where steering command $\phi$ is given. In dashed box 3, the MechaCell device reaches to the end of the path, however due to the cyclic feeding of our reference algorithm the device tries the next B-shape before the experiment is stopped. This extra movement can also be seen in Fig. 14. Deviation from path error parameter's value is in sub-centimeter scale, which is a successful path tracking for out requirements. 

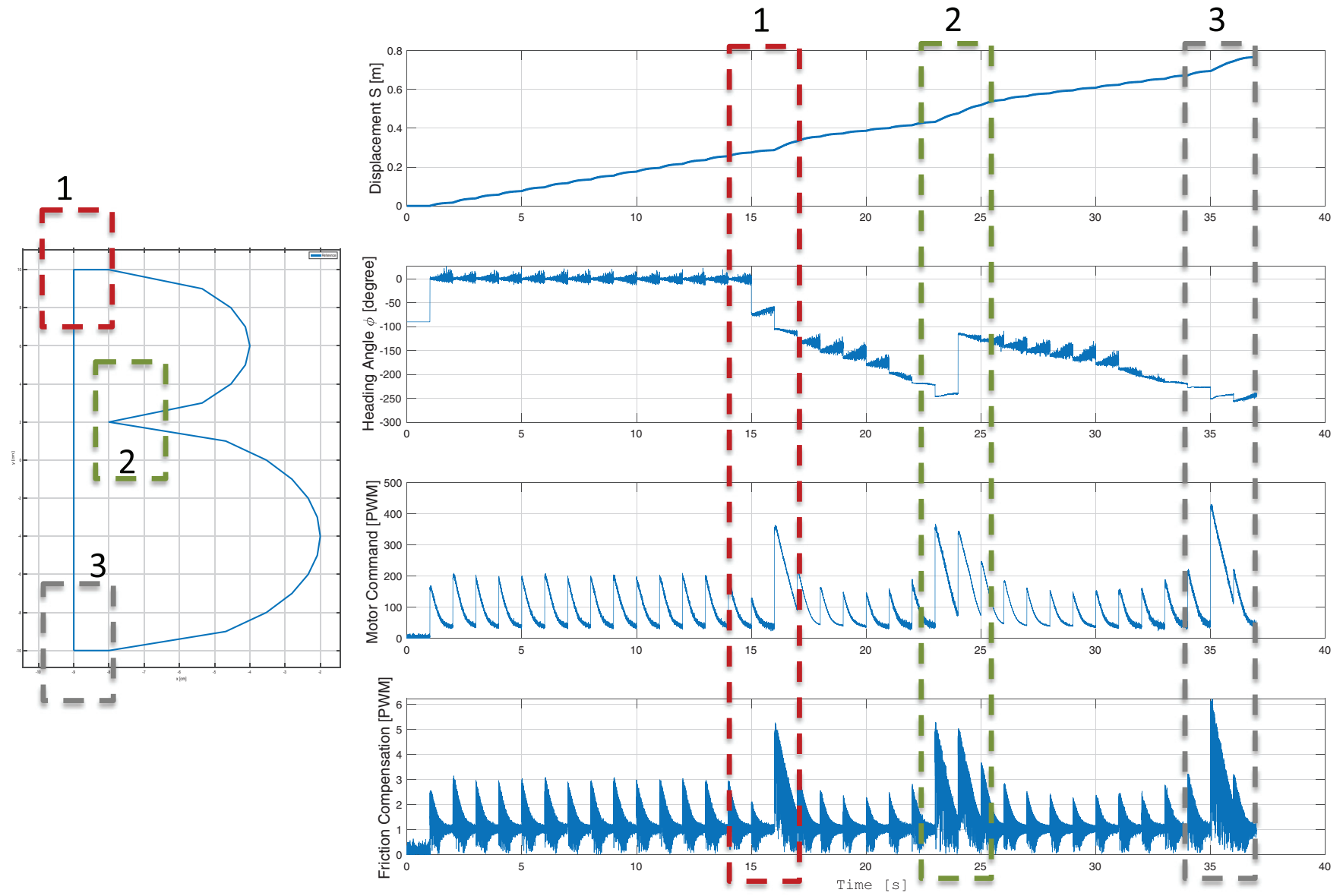

1 2

Fig. 15. Results of the path following tasks of a single MechaCell with experiments and simulation.

\section{Conclusion}

Work presented in this paper outlines the motion controller design of a modular mechatronic device, the MechaCell. MechaCell is simple module that contains sensor, actuator and control features and as a pack, MechaCells may able to overcome complicated tasks such as workpiece manipulation on a surface. A scalable and novel locomotion system is integrated in the design on the MechaCell that uses a mechanism that converts steerable unbalanced mass motion into controller and precise translational motion.

The motion controller features three sub-systems: a translational controller (s-controller), steering and orientation controllers ( $\phi$ and $\gamma$ controllers). The controller uses an improved friction compensation algorithm with corrected inertial effects that linearizes the plant dynamics. Friction force is estimated using the mathematical model developed for the MechaCell. A popular friction estimation approach, LuGre friction model, is used and this method is extended so that rapid changes in the direction of motion is also compensated. Moreover, the estimation also uses the inertial acceleration of the unbalanced mass by considering motion of the parts of the device. Our simulation and experimental results show the designed translation system (controller and the mechanism) works well including the extension on the friction algorithm which provides additional improvements up to $14 \%$ for contour tracking error as compared to the results given in [14] that was obtained using PID controllers, estimation based on Coulomb friction and relative acceleration of the unbalanced mass only. We believe current results are a good step in the right direction for precise motion applications although still in its early stages. However, there may some applications in the near future, for example, to repair the surface of hard to reach surfaces by additive manufacturing or laser sintering of fragile surfaces where the precision requirements can be more forgiving.
Future work will include, improving the orientation $(\gamma-)$ control using detailed plant dynamics, miniaturization of the MechaCell device translation module and implementation of this system with multiple MechaCells forming a pack to perform a precision task as positioning for low impact manufacturing devices. Communication delay is not studied in this work and is a factor when more than one devices are controlled together as part of a pack.

\section{Declaration of Competing Interest}

The authors declare that they have no known competing financial interests or personal relationships that could have appeared to influence the work reported in this paper.

\section{Acknowledgments}

Research supported by European Commission through Seventh Framework Program for Research and Technological Development under the contract PIRG07-GA-2010-268420.

\section{References}

[1] Cakmakci M, Ulsoy AG. Improving component-swapping modularity using bidirectional communication in networked control systems. IEEE/ASME Trans Mechatron 2009;14(3):307-16. doi:10.1109/TMECH.2008.2011898.

[2] Cakmakci M, Ulsoy AG. Swappable distributed MIMO controller for a VCT engine. IEEE Trans Control Syst Technol 2011;19(5):1168-77. doi:10.1109/ TCST.2010.2080275.

[3] Grosu V, Rodriguez-Guerrero C, Grosu S, Vanderborght B, Lefeber D, et al. Design of smart modular variable stiffness actuators for robotic assistive devices. IEEE/ASME Trans Mechatron 2017. doi:10.1109/TMECH.2017.2704665. 1-1

[4] Suryadevara NK, Mukhopadhyay SC, Kelly SDT, Gill SPS, et al. WSN-based Smart sensors and actuator for power management in intelligent buildings. IEEE/ASME Trans Mechatron 2015;20(2):564-71. doi:10.1109/TMECH.2014.2301716. 
[5] Ulrich K, Tung K. Fundamentals of product modularity. New York: ASME; 1991. p. 73-9.

[6] AlGeddawy T. A DSM cladistics model for product family architecture design. Procedia CIRP 2014;21:87-92. doi:10.1016/j.procir.2014.03.122.

[7] Yim M, Shen W-m, Salemi B, Rus D, Moll M, Lipson H, Klavins E, Chirikjian G, et al. Modular self-reconfigurable robot systems [grand challenges of robotics]. IEEE Robot Autom Mag 2007;14(1):43-52. doi:10.1109/MRA.2007.339623.

[8] Kamimura A, Kurokawa H, Yoshida E, Murata S, Tomita K, Kokaji S, et al. Automatic locomotion design and experiments for a modular robotic system. IEEE/ASME Trans Mechatron 2005;10(3):314-25. doi:10.1109/TMECH.2005.848299.

[9] Wei H, Cai Y, Li H, Li D, Wang T, et al. Sambot: a self-assembly modular robot for swarm robot. In: 2010 IEEE international conference on robotics and automation. IEEE; 2010. p. 66-71. doi:10.1109/ROBOT.2010.5509214.

[10] Piranda B, Laurent GJ, Bourgeois J, Clévy C, Möbes S, Fort-Piat NL, et al. A new concept of planar self-reconfigurable modular robot for conveying microparts. Mechatronics 2013;23(7):906-15. doi:10.1016/j.mechatronics.2013.08.009.

[11] Rubenstein M, Ahler C, Nagpal R. Kilobot: A low cost scalable robot system for collective behaviors. In: 2012 IEEE international conference on robotics and automation. IEEE; 2012. p. 3293-8. doi:10.1109/ICRA.2012.6224638.

[12] Caprari G., Balmer P., Piguet R., Siegwart R., et al. The autonomous micro robot "Alice": a platform for scientific and commercial applications. In: MHA'98. proceedings of the 1998 international symposium on micromechatronics and human science. - creation of new industry - (Cat. No.98TH8388). IEEE. 231-235. doi:10.1109/MHS.1998.745787.

[13] Cakmakci M., Ristevski S. A motion system. 2018. https://patents.google. com/patent/US20170066134A1/en.

[14] Ristevski S, Cakmakci M. Mechanical design and position control of a modular mechatronic device (MechaCell). In: 2015 IEEE international conference on advanced intelligent mechatronics (AIM). IEEE; 2015. p. 725-30. doi:10.1109/AIM.2015.7222623. vol. 2015-Augus

[15] Ristevski S., Cakmakci M. Mathematical model for coordinated motion of modular mechatronic devices (mechacells). In: Volume 2: diagnostics and detection; drilling; dynamics and control of wind energy systems; energy harvesting; estimation and identification; flexible and smart structure control; fuels cells/energy storage; human robot interaction; HVAC building energy M. ASME. 2015, p. V002T34A010. 10.1115/DSCC2015-9896.

[16] Dolcini PJ, Canudas de Wit C, Béchart H. Dry clutch control for automotive applications. London: Springer; 2010. doi:10.1007/978-1-84996-068-7.

[17] Armstrong-Helouvry B. Control of machines with friction, 128. Springer; 1991. http://www.springer. com/gp/book/9780792391333\#otherversion=978146 1367741.

[18] Astrom KJ, Canudas-de Wit C. Revisiting the lugre friction model. IEEE Control Syst 2008;28(6):101-14. doi:10.1109/MCS.2008.929425.
[19] Lee TH, Tan KK, Huang S. Adaptive friction compensation with a dynamical friction model. IEEE/ASME Trans Mechatron 2011;16(1):133-40. doi:10.1109/ TMECH.2009.2036994.

[20] Ruderman M. Mechatronics presliding hysteresis damping of LuGre and Maxwell-slip friction models. Mechatronics 2015;30:225-30. doi:10.1016/ j.mechatronics.2015.07.007.

[21] Keck A, Zimmermann J, Sawodny O. Friction parameter identification and compensation using the elastoplastic friction model. Mechatronics 2017;47:168-82. doi:10.1016/j.mechatronics.2017.02.009.

[22] Tustin A. The effects of backlash and of speed-dependent friction on the stability of closed-cycle control systems. J Inst Electr Eng- Part IIA 1947;94(1):143-51. doi:10.1049/ji-2a.1947.0021.

[23] Holmes DG, Lipo TA. Pulse width modulation for power converters : principles and practice. John Wiley; 2003. http://cds.cern.ch/record/1480869.

[24] Freidovich L, Robertsson A, Shiriaev A, Johansson R, et al. LuGre-model-based friction compensation. IEEE Trans Control Syst Technol 2010;18(1):194-200. doi:10.1109/TCST.2008.2010501.

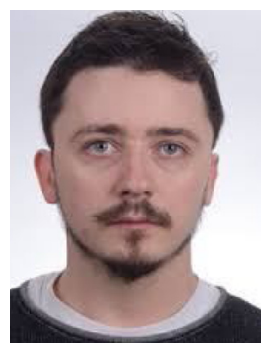

Stefan Ristevski was a graduate student at Bilkent University Mechanical Engineering Department where he received his master's degree. After spending some time in the industry gaining experience in real-time control systems and HMIs, he is currently a Ph.D. Candidate at University of South Florida.

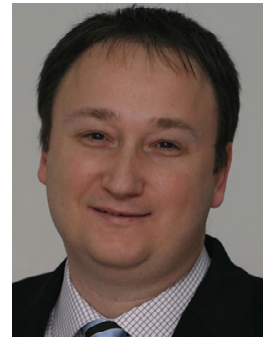

Melih Cakmakci is an Assistant Professor of Mechanical Engineering at Bilkent University in Ankara, Turkey. He received his B.S degree in Mechanical Engineering from M.E.T.U Ankara in 1997. He received his M.S and Ph.D. in Mechanical Engineering Degrees from University of Michigan in 1999 and 2009 respectively. His research areas include modeling, analysis and control of dynamic systems, Prior to joining Bilkent University, he was a senior engineer at the Ford Scientific Research Center at Dearborn, Michigan, USA. He is a member of ASME, IEEE and SAE. 